\title{
Inflation rule for Gummelt coverings with decorated decagons and its implication to quasi-unit-cell models
}

\author{
Hyeong-Chai Jeong \\ Department of Physics, Sejong University, \\ Kwangjin-ku, Seoul 143-747, Korea
}

\begin{abstract}
The equivalence between quasi-unit-cell models and Penrose-tile models on the level of decorations is proved using inflation rules for Gummelt coverings with decorated decagons. Due to overlaps, Gummelt arrangement of decorated decagons gives rise to nine different (context-dependent) decagon decorations in the covering. The inflation rules for decagons for each of nine types are presented and shown that inflations from differently typed decagons always produce different decorations of inflated decagons. However, if the original decagon region is divided into "equivalent" rhombus Penrose tiles, typed-decagon arrangements in the tiles (of the same shape) become identical for the fourfold inflated decagons. This implies that a decagonal quasi-unit-cell model can be reinterpreted as a Penrose-tile model with fourfold deflated super-tiles.
\end{abstract}

\section{INTRODUCTION}

Quasicrystals are solids exhibiting long-range translational order with a rotational symmetry that is forbidden in a periodic system [1]. They have a quasiperiodic translational order and their lattice structures are called quasicrystalline lattices or quasilattices. There were several different ways of obtaining a quasilattice. Projection, dual grid, inflation, Penrose matching-rule and Gummelt overlapping-rule methods are some of known examples of obtaining a quasilattice 2, 3].

The well-known two-dimensional (2D) Penrose lattice can be obtained by each of above methods. All of them make the equivalent Penrose lattice structure but the basic building blocks they use to produce the structure are not the same. For example, the 5D hypercube, the two types of rhombic tiles and the decagon are the basic building blocks in the projection, arrow matchingrule and Gummelt overlapping-rule methods respectively. Therefore, the different ways of obtaining (the same) quasilattice structures may produce different sets of atomic models, which are obtained by decorating each of basic building blocks identically and applying the operations to get the quasilattice structure. Therefore, equivalence in concept between two different approaches for quasicrystals should be investigated on two different levels, on the level of the lattice structures and on the level of atomic models.

Recently, we considered the relationship between rhombus-Penrose-tile (RPT) models, which are based on the arrow matching-rule method, and decagonal quasi-unit-cell (dQUC) models, which are based on the Gummelt overlapping-rule method 4]. An RPT model corresponds to decorating each fat rhombus identically and each skinny rhombus identically, and joining them to form a Penrose tiling. Similarly, a dQUC model corresponds to decorating decagons identically and then covering the plane according to the Gummelt overlapping rule. The decagon is called a quasi-unit cell (QUC) since its role corresponds to the role of unit cell in a periodic crystal. It is similar to the unit cell in the sense that the QUC is a single repeating unit whose decoration determines the entire atomic structure of the solid. However, unlike the unit-cells, it overlaps its neighbors. Because of the overlaps, Gummelt arrangement of identically decorated decagons can produce the contextdependent decoration of decagons in the covering. Therefore, the equivalence between Penrose tiling and Gummelt Covering on the level of lattice [3, 5, [6] may not guarantee the equivalence between RPT-models and dQUC models.

The lattice structure for real quasicrystals are often obtained by the 3D generalization of the planar Penrose lattice. For example, the structure of decagonal quasicrystals can be considered as a periodic stack of decagonal quasicrystalline planes. Therefore, the basic building blocks for real materials should be $3 \mathrm{D}$ also. For decagonal quasicrystals, they are two types of rhombic prisms for an RPT model 81 and the decagonal prisms for a dQUC model [9]. Recently, we have constructed a dQUC model with the decagonal prism as the basic building block for $\mathrm{Al}_{72} \mathrm{Ni}_{20} \mathrm{Co}_{8}$, one of the best-characterized quasicrystals [9, 10]. With the dQUC model, we could successfully reproduce the observed HRTEM images of $\mathrm{Al}_{72} \mathrm{Ni}_{20} \mathrm{Co}_{8}$, as well as its measured stoichiometry, density and symmetry. Very recent experiments on the surface images of the above decagonal quasicrystals 11] are also explained more naturally with dQUC approaches than with conventional RPT models. Furthermore, dQUC models provide simpler theoretical explana- 
tions for the existence of quasicrystals [5]. For RPT models, two or more clusters analogous to Penrose tiles are needed and the complex atomic interactions are required to mimic the arrow matching rules while the dQUC models use only a single type of basic building blocks as for crystals. Therefore, dQUC models seem to provide physically more natural explanation for the quasicrystal formation and structures.

The mathematical questions on the relationship between the set of dQUC models and the set of conventional atomic models, such as RPT models or hyper-cubic decoration models, are not fully investigated yet. In our recent paper, we showed that an RPT model is a dQUC model with the same edge size but the converse is not true [4]. Some dQUC models cannot be interpreted as RPT models with the same edge length. However, we conjectured that a dQUC model can be an RPT model with different edge size. Here, we provide a mathematical proof for this claim. We first present the inflation rule for a dQUC model and show that a dQUC model is an RPT model with the super-tiles whose edge is $\tau^{4}$ times longer than the edge of the quasi-unit decagon, where $\tau=\frac{1+\sqrt{5}}{2}$.

\section{INFLATION RULE FOR DECORATED DECAGONS}

Real quasicrystals are 3D and therefore the basic building block for a dQUC model for real materials should be a decagonal prism. However, here we mod out the periodic direction and take a 2D regular decagon as the basic building block. The 2D Gummelt covering then represents the lattice structure of each quasicrystalline plane and the atomic decoration of the basic decagon is obtained by the projection of atoms in the basic decagonal prism of the model for a real quasicrystal. For a classification of full 3D patterns from Gummelt prism decoration, see, Lord and Ranganathan 12].

In 1996, Gummelt showed that a 2D quasicrystalline structure with the decagonal symmetry can be obtained when the decagons, shown in Fig. 1(a), are arranged with a specific overlapping rule [3]. The overlapping rule demands that two decagons may overlap only if the shaded regions overlap. An infinite arrangement of decagons according to the Gummelt overlapping rule is called a Gummelt covering. If we replace every decagon in the covering by a Jack 13] as described in Fig. 1(b), we obtain a Penrose tiling with rhombi whose edge length is the same as that of the decagon as shown in solid lines in Fig. 1(c). Due to the self-similar properties of the Gummelt covering, we can obtain

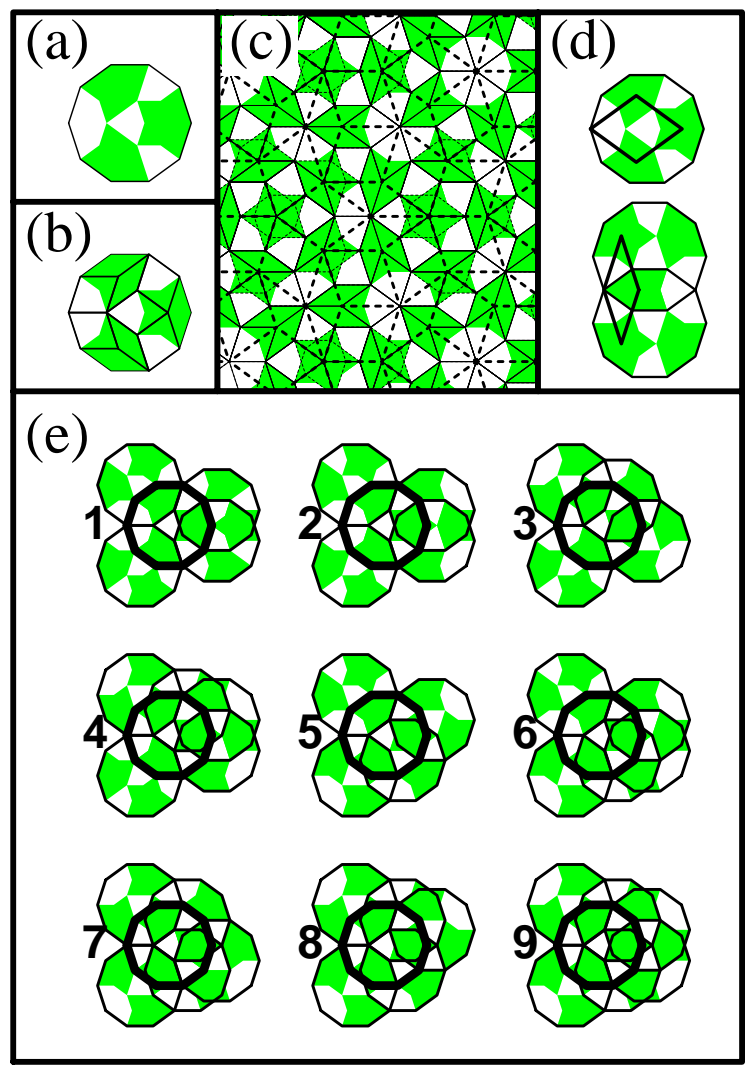

FIG. 1: (a) A marked regular decagon, the basic building block for a Gummelt covering. The decagon is marked with shaded to represent the Gummelt overlapping rule. In the overlapped region between two neighboring decagons, shaded part from one decagon should be also shaded from the other decagon. (b) Jack inscribed in a decagon. (c) A Gummelt covering can be mapped into a Penrose tiling by dividing up central area of decagon into Jack (thin solid lines). A deflated Penrose tiling (thick dashed lines) is obtained if we place a deflated skinny tile on every pair of decagons and replace each decagon by a deflated fat tile as show in (d). (e) Nine ways of "surrounding" a decagon appeared in a Gummelt covering. The centered decagon (thick lines) in the $k$-th $(k=1, \ldots, 9)$ surrounding configurations is called a type- $k$ decagon.

a Penrose tiling with other scale from the same covering. For example, if we place a skinny tile on every pair of decagons and replace each decagon by a fat tile as shown in Fig. 1(d), we get another Penrose tiling with "deflated" (bigger) tiles as shown in thick dashed lines in Fig. 1(c).

The mathematical equivalence between the lattice structures of the Penrose tiling and the Gummelt covering can be shown by considering the nearest-neighbor configurations of decagons allowed by the overlapping rules [ 6 . There are 20 
different ways of surrounding a decagon with neighboring decagons, where surrounding a decagon means the edges of the decagon is covered by the interior of neighboring decagons. These 20 configurations are equivalent to the configurations of "surrounding" a fat rhombus with arrow edged rhombus tiles [6]. Among these 20 configurations of surrounding decagons, only 9 configurations, shown in Fig. 1(e), appear in a Gummelt covering. The centered decagon in the $k$-th surrounding configurations of Fig. 1(e) is called a type- $k$ decagon where $k=1, \cdots, 9$.

Recall that a dQUC model is defined by an atomic arrangement resulted from the covering of identically decorated decagons which are arranged according to the Gummelt overlapping rule. Although we arrange the identically decorated decagons, differently-typed (or different context) decagons can be decorated differently in the covering since they have different overlaps with the neighboring decagons.

A Gummelt covering is self-similar as a Penrose tiling is and therefore allows an inflation operation. The "inflation of a decagon" is a transformation in which a decagon is replaced by 5 decagons as shown in Fig. 2(a). The edges of inflated decagons are $1 / \tau$ times smaller than those of the original decagon. The letters A, $\cdots, \mathrm{E}$ represent the positions of the inflated decagons. An infinite number of iterations of inflations (together with rescaling in the length by $\tau$ ) produce a Gummelt covering. Inflation of a decagon covering means that the inflation of every decagon in the entire covering. Inflation of a Gummelt covering produces another Gummelt covering.

The types of inflated decagons are determined by the type of the original decagon. The inflated decagon at the position A is always type- 1 by the definition of the inflation. The types of the inflated decagons at the other 4 positions depend on the type of original decagon. Unless the original decagon is type-1, they are uniquely determined for a given type of the original decagon. Note that a typed decagon implies a finite number of decagons at fixed neighboring positions. At least, the $k$-th surrounding configuration (Fig. 1(e)) is always implied to the type- $k$ decagon as the "nearest neighbors". In addition to this surrounding decagons, "accompanied decagons" as the "next nearest neighbors" are also implied for most cases (except type-1). "Accompanied decagons" of the configuration- $k$ are the decagons which are forced to be there in a Gummelt covering for the given configuration- $k$. Figure 2(b) shows the case of the type-9 decagon. Six decagons drawn with thin solid lines are the surrounding decagons of the type-9 decagon. Two decagons (dotted-line)
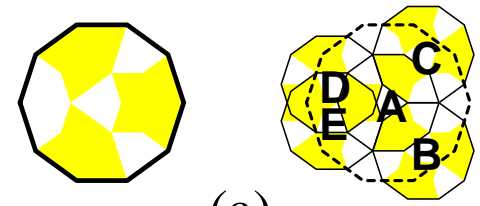

(a)

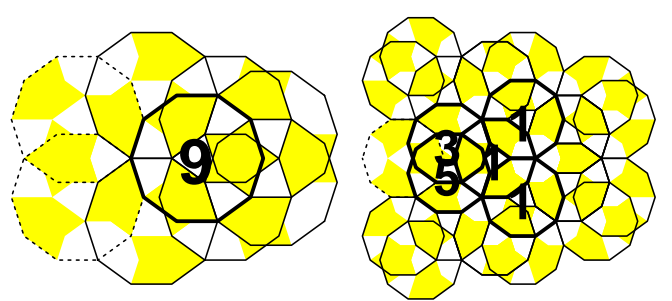

(b)
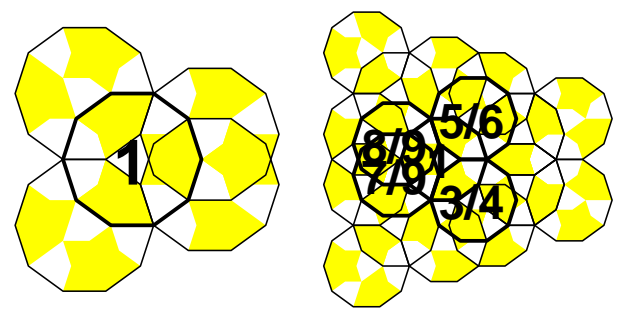

(c)

FIG. 2: (a) Inflation of a decagon is defined as replacement of a decagon (left) to the 5 smaller decagons shown at the right. The letters $\mathrm{A}, \cdots, \mathrm{E}$ at the centers of the inflated decagons are shown for later references. (b) Types of inflated decagons for a type-9 decagon. Inflations of surrounding (thin solid lines) and accompanied (dotted lines) decagons determine the types of the inflated decagons. The types of the inflated decagons at the position A,B,C,D, and E are 1,1,1,3, and 5 respectively. (c) Types of inflated decagons for a type-1 decagon. In this case, there are no accompanied decagons. Inflations of surrounding decagons are not enough for unique determination of the inflated decagon types. In addition to the position of 4 surrounding neighboring decagons, positions of 6 next-nearest-neighboring decagons are needed to fix the types of inflated decagons. See Fig. 3.

at the left of the figure are some of accompanied decagons. The inflated decagons of the type- 9 decagon (thick solid line decagons at the right figure of Fig. 2(b)) are fully surrounded by the inflated decagons of the surrounding (thin solid lines) and accompanied (dotted line) decagons of the original type-9 decagon. Inflations of surrounding 6 decagons force the inflated decagons at the positions $\mathrm{B}$ and $\mathrm{C}$ to be type-1. The inflated decagons at the positions $\mathrm{D}$ and $\mathrm{E}$ are not fully surrounded by the inflated decagons (solid lines) of the original surrounding decagons. However, inflations of the accompanied decagons of the configuration-9 
decagon give rise to the inflated decagon (dottedline) at the left and the $\mathrm{D}$ and $\mathrm{E}$ inflated decagons become type-3 and 5 respectively. Similar mechanisms determine the types of inflated decagons uniquely for the type- $2, \cdots$,type- 9 decagons and their types are given at table 1. (Subtypes of the type- 1 decagons $\left(1_{\mathrm{fss}}, \cdots, 1_{\mathrm{fff}}\right)$ in the table are explained later.)

Table I. Types of Inflated decagons

\begin{tabular}{|c|c|c|c|c|c|c|}
\hline \multirow{2}{*}{\multicolumn{2}{|c|}{\begin{tabular}{|c|} 
Decagon \\
Type
\end{tabular}}} & \multicolumn{5}{|c|}{ Inflated decagons } \\
\hline & & $\bar{A}$ & $\bar{B}$ & $\mathrm{C}$ & $\overline{\mathrm{D}}$ & $\mathrm{E}$ \\
\hline \multirow{13}{*}{1} & $\overline{1_{\text {SSS }}}$ & $1_{\mathrm{SSS}}$ & $\overline{3}$ & $\overline{5}$ & $\overline{88}$ & $\overline{7}$ \\
\hline & $1_{\mathrm{fSS}}$ & $1_{\mathrm{SSS}}$ & 3 & 5 & 9 & 9 \\
\hline & $1_{\mathrm{fsf}}$ & $1_{\mathrm{SSS}}$ & 4 & 5 & $\overline{9}$ & $\overline{9}$ \\
\hline & $1_{\text {ffs }}$ & $1_{\mathrm{SSS}}$ & 3 & 6 & 9 & 9 \\
\hline & $1_{\mathrm{fff}}$ & $1_{\mathrm{SSS}}$ & 4 & 6 & 9 & 9 \\
\hline & 2 & $1_{\mathrm{fSS}}$ & 2 & 2 & 9 & 9 \\
\hline & 3 & $1_{\mathrm{fsf}}$ & 2 & $1_{\text {ffs }}$ & 4 & 8 \\
\hline & 4 & $1_{\mathrm{ffs}}$ & 2 & $1_{\text {fff }}$ & 4 & 8 \\
\hline & 5 & $1_{\mathrm{ffs}}$ & $1_{\mathrm{fsf}_{\mathrm{s}}}$ & 2 & 7 & 6 \\
\hline & 6 & $1_{\mathrm{ffs}}$ & $1_{\mathrm{fff}}$ & 2 & 7 & 6 \\
\hline & 7 & $1_{\text {fff }}$ & $1_{\text {fff }}$ & $1_{\text {ffs }}$ & 3 & 5 \\
\hline & 8 & $1_{\mathrm{fff}}$ & $1_{\mathrm{fsf}}$ & $1_{\mathrm{fff}}$ & 3 & 5 \\
\hline & 9 & $1_{\mathrm{fff}}$ & $1_{\mathrm{fff}}$ & $1_{\mathrm{fff}}$ & 3 & 5 \\
\hline
\end{tabular}

This is not the case for the type-1 decagon. Unlike the other types, there are no accompanied decagons at the next nearest neighbors for the type-1 decagon. As shown in Fig. 22 (c), there are 4 surrounding decagons in this case and their inflations are not enough for the unique determination of the inflated decagon types. Note that the configuration-1 in Fig. 1(e) corresponds to a Jack configuration with deflated tiles if a deflated fat rhombus is inscribed in each decagon as in Fig. 3(a) 6]. In a Penrose tiling, a Jack configuration is always a part of the $\mathrm{C}^{\prime}$-decagon [6, 7] consists of a Jack and three hexagons as shown in Fig. [3(b). There are two possible configurations for filling each hexagon, $s$ and $f$ orientation. Each hexagon consists of three tiles, one skinny tile and two fat tiles and the skinny tile in an $s[f]$-oriented hexagon is at the side near [far from] the center of the $\mathrm{C}^{\prime}$-decagon. This gives rise to 8 subtypes for the type- 1 decagon; from $1_{\text {SSS }}$ to $1_{\mathrm{fff}}$. The first, second, and third subscripts represent the orientations of the front, bottom, and top hexagons respectively. Fig. B(c) and (d) shows examples of subtyped decagons, $1_{\mathrm{Sss}}(\mathrm{c})$ and $1_{\text {fsf }}(\mathrm{d})$. All eight configurations satisfy the Gummelt overlapping rule locally but the subtype $1_{\text {sfs }}$, $1_{\text {ssf }}$, and $1_{\text {sff }}$ decagons never appear in a Gummelt covering since adding decagons to these configurations forces a violation of the overlapping rule somewhere. The subtypes of the type- 1 decagon

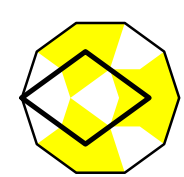

(a)

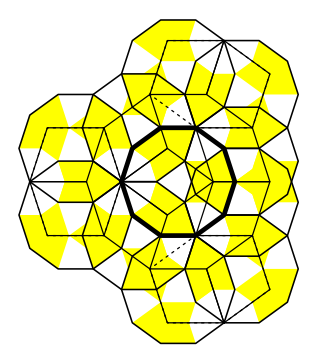

(c)

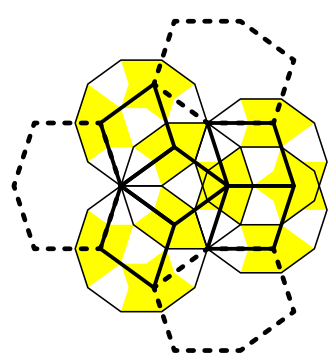

(b)

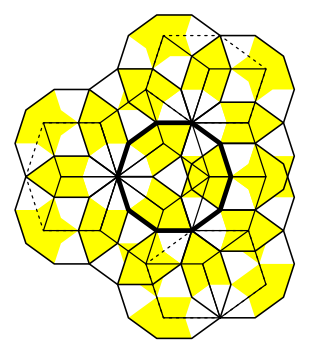

(d)
FIG. 3: (a) Deflated fat rhombus inscribed in a decagon. (b) $\mathrm{C}^{\prime}$-decagon consists of a Jack and three hexagons of deflated tiles. A hexagon is composed of two fat rhombi and one skinny rhombus. Depending on the orientation of the hexagons, type- 1 decagons are further classified by 8 subtypes $\left(1_{\mathrm{Sss}}, \ldots, 1_{\mathrm{fff}}\right)$. (c) Subtype $1_{\text {sss }}$ decagon. (d) Subtype $1_{\text {fsf }}$ decagon.

fix the positions of the 6 next-nearest-neighboring decagons (in the three hexagons) in addition to the 4 surrounding decagons and their inflations determine the types of the inflated decagons uniquely.

Table 1 shows the types of the inflated decagons for each type of the original decagon. The letters $\mathrm{A}, \cdots, \mathrm{E}$ denote the inflated decagons at the different positions denoted by Fig. 22(a).

\section{RELATIONSHIP BETWEEN DQUC MODELS AND RPT MODELS}

In this section, we compare the set of RPT models with the set of dQUC models and show that two sets are the same. It is quite easy to show that the set of dQUC models includes the set of RPT models. We can easily construct an equivalent dQUC model for a given RPT model. Recall that a Gummelt covering is obtained when we replace each Jack in a Penrose tiling by a decagon as illustrated in Fig. 1(c). For a given RPT model, by inscribing the "Jack decoration" (obtained by a Jack configuration of the decorated tiles of the RPT model) to the decagon, an equivalent dQUC model is obtained [4] as illustrated in Fig. [4(a) and 


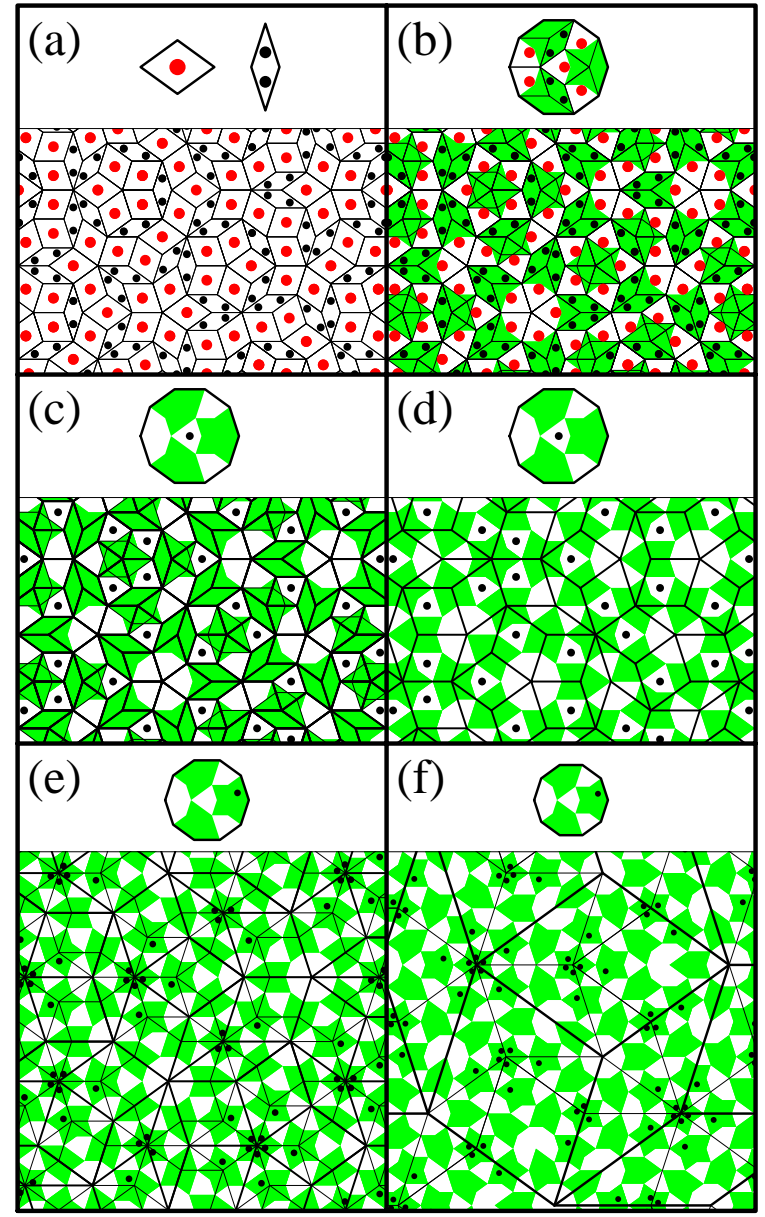

FIG. 4: Relationship between dQUC models and RPT models. An RPT atomic model is obtained by decorating each fat rhombus by one atom and each skinny rhombus by two atoms as shown in the upper panel of (a) and joining them to form a Penrose tiling. The RPT model of (a) is a dQUC model since the same atomic model can be obtained by decorating the decagon by 9 atoms as shown in the upper panel of (b) and arrange them to form the Gummelt covering. The dQUC model of (c) is not an RPT model with the same edge size since some fat rhombi have atoms while the others don't. However, it is an RPT model with the singly deflated Penrose tiles as illustrated in (d). For the dQUC model of (e), the singly deflated fat rhombi (thin lines) are not decorated identically. Neither doubly (thick lines (e)) nor triply (thin lines in (f)) deflated rhombus tiles are decorated identically. However, it is an RPT model with fourfold deflated rhombus tiles as shown by thick lines (for clarity, only few super-tiles are shown).

(b). The atomic arrangement resulted from the Penrose arrangement of the decorated tiles in (a) is identical to that results from the covering of the decorated decagons in (b).

However, it is not obvious if there is always an
RPT model which is equivalent to a given dQUC model. Here, we show that it is the case by explicitly constructing such an RPT model using the inflation rule for decorated decagons.

Before we discuss the relationship between the set of all RPT models and that of all dQUC models, let us first consider restricted sets of models whose basic building blocks (tiles or decagon) have the same edge length. As mentioned before, the length of the decagon edges of the dQUC model, obtained by the "Jack decoration" of an RPT model, is the same as the length of the tile edges. That is, for any RPT model, there is an equivalent dQUC model with the decagons whose edge length is the same as the tile edge length. However, the converse is not true. Figure 4(c) shows a counter example in which a decagon is decorated with an atom at the center. When the decagons in the coverings are resolved into a Jack, some fat rhombi have atoms whereas some others do not. Yet, it is an RPT model with the (singly) deflated Penrose tiles as illustrated in Fig. 4(d). Each deflated fat rhombus is decorated identically (with an atom) and so is each deflated skinny rhombus (with no atom). However, this is rather accidental case for the decagon decoration of Fig. 4(c). For the example of Fig. 4(e), the singly deflated fat rhombi (thin lines) are decorated with zero, one or two atoms depending on the context. Neither doubly (thick lines in Fig. 廿(e)) nor triply (thin lines in Fig. 4(f)) deflated rhombus tiles are decorated identically illustrated. For example, a doubly deflated fat tile has 1 or 2 atoms and a triply deflated fat tile has 5 or 6 atoms depending on the context. Therefore, in general, a dQUC model is not an RPT model with tiles up to triply deflated tiles. However, the dQUC model of Fig. प(e) is an RPT model with fourfold deflated rhombus tiles as shown in the thick lines in Fig. 4(f). The (fourfold-deflated) super-tiles of the same shapes are decorated identically. (for clarity, only few super-tiles are shown here but we could not find any super-tiles decorated differently even in much larger sample). Now, the question is whether this is also accidental result for the example of (e) or true for a general atomic decorations.

Below, we show that a dQUC model is an RPT model with the (fourfold-deflated) super-tiles in general. Note that the decagons with the same type are decorated identically even in the covering since their surrounding decagon arrangements are the same. Therefore, deflated tiles are decorated identically if the types of the decagons at the same positions in all deflated tiles are the same. Therefore, we now consider the possible decagon types in the deflated tiles and see if they are unique. Let us begin with singly deflated tiles. We know that 


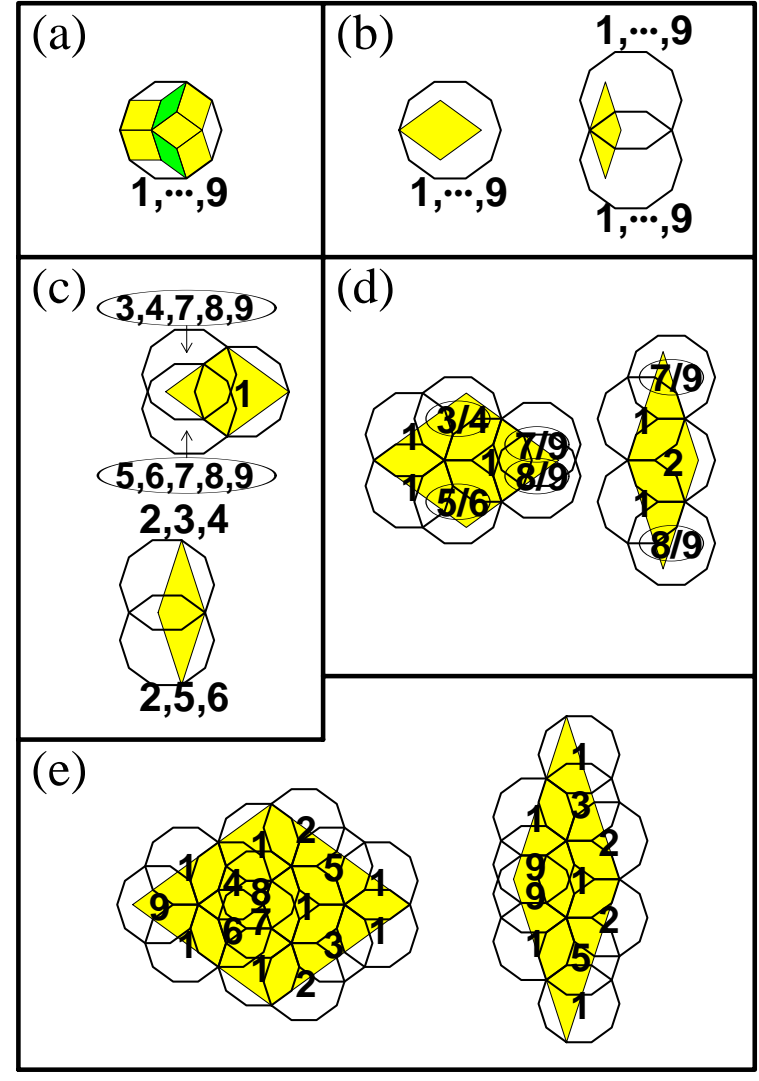

FIG. 5: Deflated Penrose tiles and typed decagons. The numbers at the center of or near the decagons represent their decagon types. The positions and types of the decagons in the deflated tiles are determined by the inflation rule of dQUC. (a) The original rhombi and a decagon with the same edge size. The rhombi can be decorated differently depending on their position in the decagon. (b) Singly deflated rhombi and typed decagons. The types of the decagons which cover the deflated rhombi are not uniquely determined. They can be any of 9 types. (c) Doubly deflated rhombi and typed decagons. The types of the decagons which cover the doubly deflated rhombi are not uniquely determined. For example, the type of the decagon which covers the lower part of the skinny shaped tile can be type-2, 5 or 6 . (d) Triply deflated rhombi and typed decagons. The types of the decagons which cover the triply deflated rhombi are not uniquely determined. For example, the type of the decagon which covers the lowest part of the skinny shaped tile can be type- 8 or 9 . (e) Fourfold deflated rhombi and typed decagons. The types of decagons are uniquely determined for both fat and skinny shapes.

a Penrose tiling with deflated tiles is obtained if we place deflated fat and skinny tiles in decagons as shown in Fig. 1(d). However, a general dQUC model cannot be an RPT model with (singly) de- flated tiles since they are not decorated identically. We already presented such an example in Fig. 4 (e). In general, singly deflated fat tile are decorated differently since the types of the decagons which cover the deflated tiles are not uniquely determined as illustrated in Fig. 5(b). A singly deflated fat tile (in the deflated tiling) is always covered by

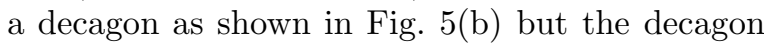
types can be any of nine types. Since the differently typed decagons are decorated differently for a general dQUC model, deflated fat tiles are decorated differently. Due to similar reasons, deflated skinny tiles are decorated differently in general. A singly deflated skinny tile is always covered by a pair of decagons by the way shown in Fig. 5 (b) but their types can be any of nine.

To investigate the relationship between multitime deflated tiles and the typed decagons, we consider deflations of decagons. Deflation of decagons is the inverse process of inflation defined by Fig. 2(a). A Gummelt covering is deflated if we substitute each configuration- 1 in the covering by a deflated decagon whose edge length is $\tau$ times larger than that of the original decagons. If each deflated decagon in the deflated covering is replaced by a Jack then a Penrose tiling with deflated tiles is obtained. If deflated decagons are replaced by doubly deflated tiles as shown in Fig. 1(d) and overlayed with the original decagons, the relationship between doubly deflated tiles and typed decagons are obtained.

Conversely, if we draw a Jack to the original decagon and inflate a decagon, relationship between the tiles (of the original size) and the inflated decagons are obtained. This relationship should be the same as the relationship between deflated tiles and decagons (of the original size) due to the self-similarity of the covering. All possible decagon types in a deflated tile is then obtained by considering all thirteen different cases in the table. The relationship between multi-time deflated tiles and the typed decagons can be obtained by inflating decagons multi times. Figures 5 (c) (e) show the relationship between (multi-time) deflated tiles and the typed decagons obtained this way. Decagon positions in the tiles of the same shape are always identical but their types are not uniquely determined for doubly ((c)) and triply $((d))$ deflated tiles. For example, doubly inflated fat tiles are covered by three decagons, decagons at the positions A, D, E of Fig. 2(a). The decagon at the position $\mathrm{D}$ can be one of the type-3,4,7,8,-9 and the decagon at the position $\mathrm{E}$ can be one of the type-5, $6,7,8,-9$ [14]. However, For the four-fold deflated tiles, the types of decagons are uniquely determined for both fat and skinny shapes as shown 
in Fig. 5(e). Therefore, any dQUC model can be interpreted as an RPT model with quadratically inflated tiles.

\section{CONCLUDING REMARKS}

We have shown that a dQUC model is an RPT model with the quadratically inflated tiles. Since an RPT model is a dQUC model, we can conclude that the set of all dQUC models is the same as the set of all RPT models. However, mathematical equivalence between two sets does not imply that they are physically equivalent in constructing atomic models for quasicrystals. Constructing dQUC models is much easier than constructing RPT models for real quasicrystals. The atomic clusters used as building blocks in dQUC models are only one kind and much smaller than the building blocks of RPT models as shown here.

Although the main contents of the paper are mathematical in nature, they can be applied in calculating some important physical quantities of dQUC models. For example, computing the density and stoichiometry of a dQUC model is complicated due to overlaps if we directly calculate them. Since an explicit way to convert a dQUC model to an RPT model is provided here, such quantities can be easily calculated using the equivalent RPT model which has no overlaps between basic building blocks.

This work was supported by Korea Research Foundation Grant (KRF-2001-041-D00062).
[1] D. Levine and P. J. Steinhardt, Phys. Rev. Lett. 53, 2477 (1984).

[2] C. Janot, Quasicrystals - A primer (Clarendon Press, Oxford, 1992).

[3] P. Gummelt, Geometriae Decdicata 62, 1 (1996).

[4] H.-C. Jeong, in Proceedings of Quasicrystals 2001 Sendai, edited by (Elsevier Science, , 2002), p. .

[5] P. J. Steinhardt and H.-C. Jeong, Nature 362, 431 (1996).

[6] H.-C. Jeong and P. J. Steinhardt, Phys. Rev. B 55, 3520 (1997).

[7] There is a one-to-one correspondence between the configurations- 1 in a Gummelt covering and the $\mathrm{C}^{\prime}$-decagons in the Penrose tiling obtained by the fat-tile decoration of Fig. 31(a). If we "deflated" a covering, a configuration-1 corresponds to a deflated decagon.

[8] H.-C. Jeong and P. J. Steinhardt, Phys. Rev. B 48, 9394 (1993).

[9] P. J. Steinhardt et al., Nature 396, 55 (1998).

[10] E. Abe et al., Phys. Rev. Lett. 84, 4609 (2000).

[11] R. McGrath, in Proceedings of Quasicrystals 2001 Sendai, edited by (Elsevier Science, , 2002), p. .

[12] E. A. Lord and S. Ranganathan, Acta Cryst. A 57, 531 (2001).

[13] M. Gardner, Sci. Am. 236, 110 (1977).

[14] This result can be directly understood from the inflation rule given at table 1 also. The columns of the inflated decagon at $\mathrm{D}$ and at $\mathrm{E}$ have type$(3,4,7,8,9)$ and type- $(5,6,7,8,9)$ respectively. However, to get the relationships between the further deflated tiles and the typed decagons the method described in the text is more straightforward. 